OPEN

SUBJECT AREAS:

VIROLOGY

EPIGENETICS

Received

3 February 2014

Accepted

8 December 2014

Published

9 January 2015

Correspondence and requests for materials should be addressed to

T.W. (tnabe@ims.utokyo.ac.jp) or M.Y. (myamagishi@mgs.k. u-tokyo.ac.jp)

\section{Epigenetic Heterogeneity in HIV-1 Latency Establishment}

\author{
Yuka Matsuda', Mie Kobayashi-Ishihara², Dai Fujikawa', Takaomi Ishida³, Toshiki Watanabe' \\ \& Makoto Yamagishi'
}

\begin{abstract}
'Laboratory of Tumor Cell Biology, Department of Medical Genome Sciences, Graduate School of Frontier Sciences, The University of Tokyo, 4-6-1 Shirokanedai, Minato-ku, Tokyo 108-8639, Japan, ${ }^{2}$ Department of Immunology, National Institute of Infectious Diseases, 1-23-1 Toyama, Shinjuku-ku, Tokyo 162-8640, Japan, ${ }^{3}$ Research Center for Asian Infectious Disease, Institute of Medical Science, The University of Tokyo, 4-6-1 Shirokanedai, Minato-ku, Tokyo 108-8639, Japan.
\end{abstract}

Despite prolonged antiretroviral therapy, HIV-1 persists as transcriptionally inactive proviruses. The HIV-1 latency remains a principal obstacle in curing AIDS. It is important to understand mechanisms by which HIV-1 latency is established to make the latent reservoir smaller. We present a molecular characterization of distinct populations at an early phase of infection. We developed an original dual-color reporter virus to monitor LTR kinetics from establishment to maintenance stage. We found that there are two ways of latency establishment i.e., by immediate silencing and slow inactivation from active infection. Histone covalent modifications, particularly polycomb repressive complex 2 (PRC2)-mediated H3K27 trimethylation, appeared to dominate viral transcription at the early phase. PRC2 also contributes to time-dependent LTR dormancy in the chronic phase of the infection. Significant differences in sensitivity against several stimuli were observed between these two distinct populations. These results will expand our understanding of heterogeneous establishment of HIV-1 latency populations.

$\mathrm{H}$ uman immunodeficiency virus type 1 (HIV-1) is a causative agent of acquired immunodeficiency syndrome (AIDS). After the discovery of HIV-1, five classes of antiretroviral drugs have been developed and a combination of antiretroviral drug treatment (ART) effectively prevents viral replication under the detectable limit ${ }^{1,2}$. However, the infected individuals should continue the ART for life because interruption in the ART results in a rapid viral rebound ${ }^{3-5}$. Despite prolonged ART, HIV-1 persists as a transcriptionally inactive provirus in some cell types and at anatomical sites, which is defined as a HIV-1 latent reservoir ${ }^{6}$. This virus reservoir is now a major obstacle for HIV-1 cure, because the ART alone cannot eradicate this population. Interestingly, detailed studies of residual viremia have shown that the rebound virus is archival and non-evolving from the virus before $\mathrm{ART}^{7-9}$. Latently infected $\mathrm{CD} 4 \mathrm{~T}$ cells harboring competent provirus are thought to be a major source of intact HIV-1.

The HIV-1 5'LTR, located at the $5^{\prime}$ end of the integrated provirus, contains the promoter and enhancer elements that accelerate HIV-1 transcription by host transcription factors, including NF- $\kappa B$, NFAT, and $\mathrm{Sp}^{10-12}$. The HIV-1-encoded regulatory protein Tat is necessary for sufficient viral transcription initiation and elongation ${ }^{13,14}$. In contrast, the integrated provirus forms a nucleosome structure that is affected by epigenetic regulation such as histone modification and DNA methylation. The regulators of histone acetylation and methylation negatively control HIV-1 transcription, leading to transcriptional latency ${ }^{15-18}$.

HIV-1 transcription is frequently silenced by epigenetic changes in the residual reservoir under $\mathrm{ART}^{6}$. Reversing latency has been proposed to eliminate latently infected cells ${ }^{19}$. Several activation strategies combined with the ART have been attempted, including cytokine-based immune activation therapy using interleuin-2 (IL2) or interferon- $\gamma\left(\right.$ IFN- $\gamma$ ) and prescriptions of epigenetic drugs ${ }^{20-24}$. In particular, inhibitors of epigenetic factors are respected as reagents to reactivate viruses in a large spectrum of reservoirs without inducing cell activation or proliferation. Recent clinical trials revealed that histone deacetylase (HDAC) inhibitors, valproic acid (VPA) and vorinostat (SAHA) could reactivate plasma viral RNA level in patients undergoing long-term ART ${ }^{25,26}$.

Much attention has been paid to define how the viral latency is maintained and how the latent viruses can be effectively reactivated. By using some latent models, many researchers have reported the molecular mechanisms contributing to the maintenance of viral gene suppression, including the host epigenetic system, Tat mutation, sequence variation in the LTR, and depletion of elongation factors ${ }^{27}$. It is important to understand the mechanisms by which HIV-1 latency is established to make the latent reservoir size smaller. However, detailed informa- 
tion on establishment of latency is limited due to technical obstacles, such as lack of biological indicators or cell surface markers to distinguish the latently infected population from the uninfected one, which makes it difficult to study the molecular mechanisms of latency establishment, and a very low frequency of the HIV-1 reservoir is estimated in actually infected individuals ${ }^{28}$. The most difficult problem is that the small reservoir population possesses the capability to cause immunodeficiency again.

In the present study, we developed an original reporter virus, enabling us to dissect the infected and uninfected populations and to monitor the LTR kinetics from establishment to maintenance step. We found two modes of infection, the immediately silenced and active infections. We also identified the molecular underpinnings of HIV-1 silencing by comparing the two distinct populations. Histone methylation, particularly polycomb repressive complex 2 (PRC2)-mediated histone H3 lysine 27 (H3K27) trimethylation, appears to affect viral transcription at the early phase of infection. In addition, PRC2 also contributes to the time-dependent repression of LTR activity in the actively infected population. These two distinct populations showed differential responses to physiological and pharmacological stimulations. Intervention of epigenetic regulation by multi-targeting of histone modifiers resulted in restoring the LTR activity in both populations.

\section{Results}

Establishment of a new HIV-1 silencing model. To evaluate the dynamics of LTR activity at the early phase of HIV-1 infection, we developed an original lentivirus vector that carries the double reporter genes, LTR-Tat-IRES-Venus-EF1 $\alpha-m R F P$ (Fig. 1a). This reporter construct contains two different genes encoding fluorescent proteins, Venus and mRFP, whose expressions are controlled by the HIV-1 LTR sequence and EF1 $\alpha$ promoter, respectively. EF1 $\alpha$ promoter activity is stable, supporting that mRFP expression can be used as an infected cell indicator (Supplementary Fig. 1a). In addition, this virus doesn't show any cytotoxicity because of the absence of viral genes except tat. We first transiently introduced the plasmids, CS-LTR-Tat-IRES-Venus-EF1 $\alpha-m R F P$ and CSLTR-empty-IRES-Venus-EF1 $\alpha-m R F P$, into 293FT cells. The expression levels of the two fluorescent proteins could be simultaneously monitored by flow cytometric analysis (Fig. 1b). Compared with the $\operatorname{Tat}(-)$ vector, the Tat $(+)$ vector induced high Venus expression, indicating that this reporter represented in vivo LTR action. Promoter interference appeared to be a little because the Venus level was directly proportional to mRFP expression.

Following this, we produced a VSV-G pseudotyped single-round reporter virus solution and transduced it in the Jurkat human $\mathrm{T}$ cell line to continuously evaluate the LTR activity after proviral integration into the host genome. The LTR activity within infected cells was defined by sequential gating beginning with FSC/SSC to select intact lymphocytes, subgating on the $\operatorname{mRFP}(+)$ and/or Venus $(+)$ population, and calculating the Venus profile (Fig. 1c). Venus expression was detected in cells with LTR-Tat-IRES-Venus-EF1 $\alpha$ $-m R F P$ virus compared with cells with Tat $(-)$ reporter virus at 3 days post-infection (dpi), suggesting that the LTR activity was immediately silenced when cell lacked the Tat protein. The mean of LTR activity reached a peak $4 \mathrm{dpi}$ and the LTR activity and Venus(+) population size gradually decreased during prolonged cultivation (Fig. 1d). We confirmed using a single-color reporter (CS-LTRtat-IRES-Venus) that the loss of LTR activity was not due to the effect of downstream EF1 $\alpha$ promoter DNA (Supplementary Figs. lb-d). The LTR activity was substantially silenced at $40 \mathrm{dpi}$. We treated this population with PMA/Ionomycin or TNF- $\alpha$ as NF- $\kappa$ B activators and detected robust Venus expression, indicating that the decrease in Venus level was caused by LTR inactivation (Fig. 1e). In addition, treatment with VPA restored the LTR activity in a dosedependent manner, resembling the latently infected HIV-1 in ART- treated individuals ${ }^{29}$ (Fig. 1f). Thus, these data demonstrated that the new reporter system could represent the physiological silencing of HIV-1 transcription. We note that a part of infected cells remained as Venus(-).

Two modes of latency establishment. We observed the establishment of HIV-1 infection in various cells. The human $\mathrm{T}$ cell lines, CCRF-CEM, SupT1, and Jurkat cells and HEK293FT cells were infected with the LTR-Tat-IRES-Venus-EF1 $\alpha-m R F P$ virus and analyzed for Venus expression within the infected population [Venus $(+)$ and/or $\operatorname{mRNP}(+)]$ at $3 \mathrm{dpi}$. We found that two major populations emerged in all the tested cell lines, one had strong Venus expression and the other showed weak or no Venus expression (Figs. 1c and 2a). Further, we examined the LTR activity in human CD4 $\mathrm{T}$ cells, primary host cells of latency, by using the highly concentrated dual-color reporter, which allowed us to analyze the LTR activity without the viral protein-dependent cytotoxicity. Spinoculation method facilitated viral entry and following integration in non-dividing primary cells. Resting CD4 $+\mathrm{T}$ cells from a healthy donor were infected with the reporter, and the Venus expression within the infected population was evaluated at $3 \mathrm{dpi}$, which is an early phase of HIV-1 infection. We found substantial population of cells expressing the latency-associated fluorescent marker (Venus(-)/ $\operatorname{mRFP}(+))$ in primary CD4 $+\mathrm{T}$ cells (Fig. $2 \mathrm{~b}$ ). $\mathrm{T}$ cell activation by anti-CD3/CD28 antibodies reduced the latently infected population.

To further characterize the distinct populations, we sorted the infected Jurkat cells by Venus/mRFP fluorescences. Each population could be sorted with a cell sorter system at $3 \mathrm{dpi}$ and was subjected to the following analyses (Fig. 3a): First, Alu-PCR analysis of genomic DNA detected similar amount of proviral DNA either in the actively or silently infected populations (Fig. 3b). Absolute quantification of Venus mRNA level demonstrated that the Venus expression pattern appeared varied due to the transcriptional activity of LTR (Fig. 3c). Indeed, transcriptional stimulation by PMA/Ionomycin or TNF- $\alpha$ treatment partially restored the LTR activity and Venus mRNA in the silent integration population (Fig. 3d).

According to previous literatures, we investigated the epigenetic status of both integrated LTR in the above sample set. HIV-1 LTR contains some $\mathrm{CpG}$ sites that contribute to transcriptional repres$\operatorname{sion}^{30,31}$. However, bisulfate DNA sequencing showed no acquisition of CpG methylation at LTR in either population (data not shown). Whereas, evaluation of covalent histone modifications by ChIP assay revealed that histone $\mathrm{H} 3$ acetylation $(\mathrm{H} 3 \mathrm{Ac})$ and $\mathrm{H} 3 \mathrm{~K} 4$ trimethylation (H3K4me3), which were active histone markers, were specifically gained at the LTR region within the Venus(+) population (Fig. 3e). In contrast, histone H3K27 trimethylation (H3K27me3), which was a general repressive histone marker, was specifically accumulated at the promoter region of LTR in the Venus(-) population (Fig. 3e). Another repressive histone mark H3K9 trimethylation (H3K9me3), which has been implicated in HIV-1 latency, was not different between the two populations. To further address the LTR silencer during immediate latency establishment, we focused on levels of the H3K27me3 marker and its specific methyltransferase complex, polycomb repressive complex 2 (PRC2). ChIP assay revealed selective occupancies of EZH2 and SUZ12, essential components of PRC2, at the LTR region within the Venus(-) population (Fig. 3f). Inhibition of PRC2 by treatment with DZNep, an inhibitor of EZH2 that was an PRC2 catalytic unit, partially reactivated the LTR activity in a dose-dependent manner, demonstrating participation of PRC2 in the immediate silencing of LTR (Fig. 3g). Of note, cotreatment with DZNep and VPA or SAHA resulted in more effective LTR transactivation, suggesting that HDACs and PRC2 cooperate in the establishment of silent integration of HIV-1. Indeed, CTDphosphorylated Pol II occupancy in LTR region was enhanced when PRC2 and HDAC were simultaneously inhibited (Fig. 3h). 


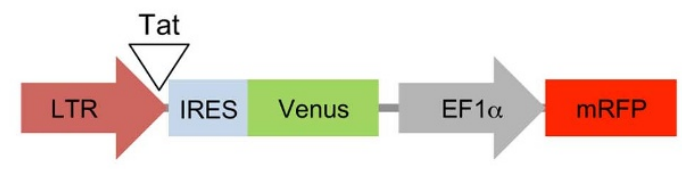

b

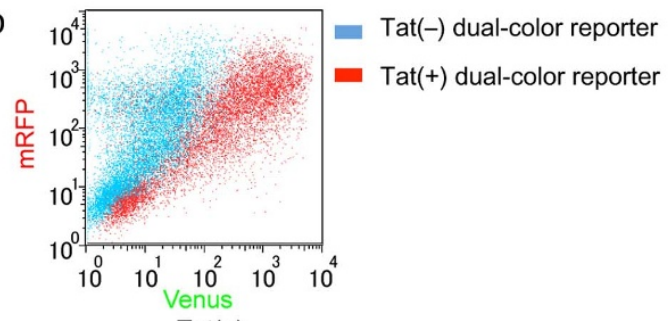

C

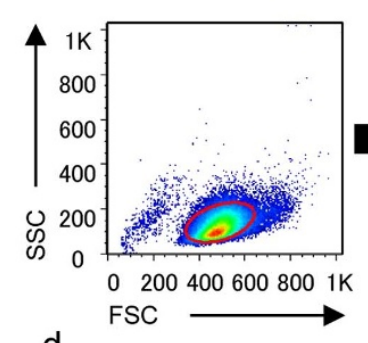

d
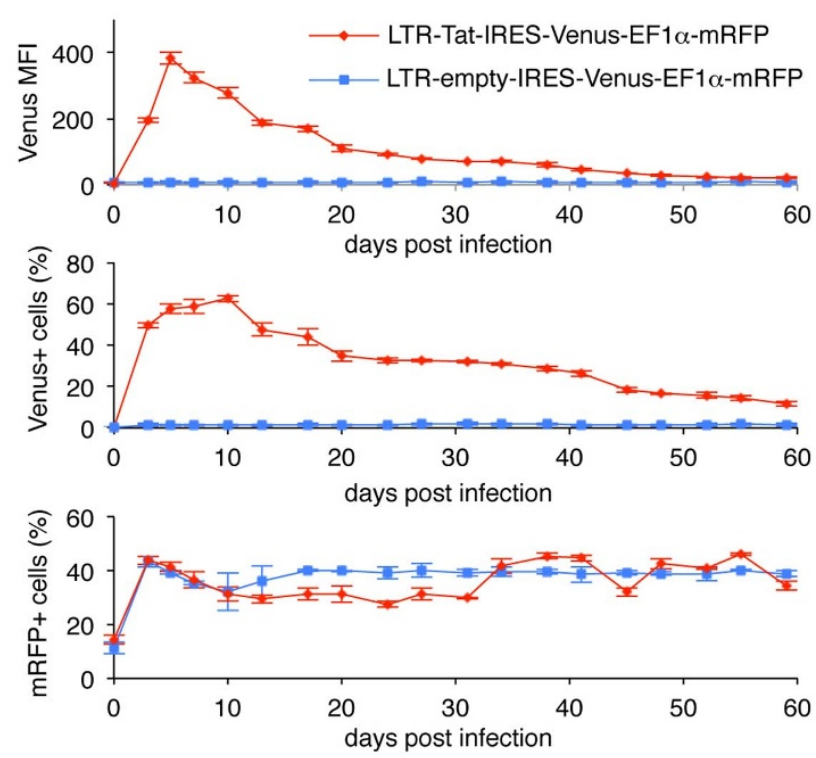

$(-) \quad$ Tat(-)
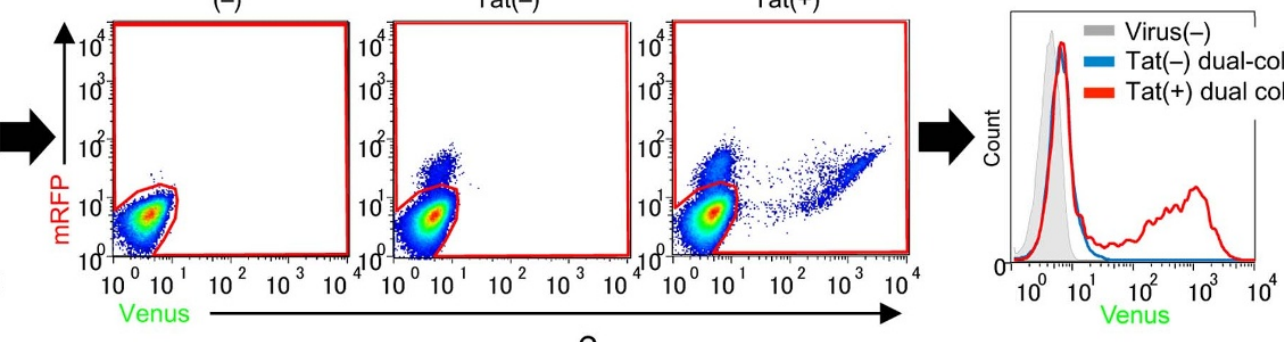

e
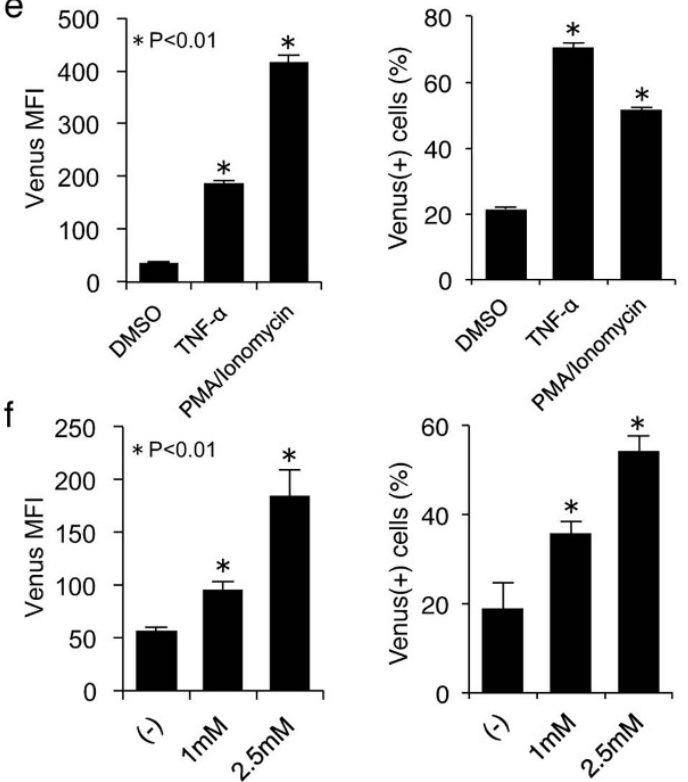

Figure 1 Establishment of new HIV-1 silencing model. (a) Construction of dual-color reporter lentivirus. Venus and mRFP expressions are controlled by LTR and EF1 $\alpha$ promoter, respectively. Tat was inserted downstream of the LTR sequence. (b) HEK293FT cells were transiently transfected with the CSLTR-Tat-IRES-Venus-EF1 $\alpha-m R F P$ or CS-LTR-empty-IRES-Venus-EF1 $\alpha-m R F P$ plasmid. The expression levels of the fluorescent proteins at 2 days post transfection were analyzed by flow cytometry. (c) Jurkat T cell line was infected with the CS-LTR-Tat-IRES-Venus-EF1 $\alpha-m R F P$ or CS-LTR-emptyIRES-Venus-EF1 $\alpha-m R F P$ lentivirus. The LTR activity within infected population was defined by sequential gating beginning with FSC/SSC to select intact lymphocytes (left scatter plot), subgating on the $\operatorname{mRFP}(+)$ and/or Venus $(+)$ population (middle scatter plots). Venus expression level within the infected population was shown in histogram plots (right). (d) Kinetic analysis within the infected population $(\mathrm{n}=3$, mean \pm SD). Upper graph shows kinetics of Venus mean fluorescence intensity (MFI); middle and lower show proportions of Venus $(+)$ and $\operatorname{mRFP}(+)$ cells $(\%)$. (e,f) The LTR-silenced total population (48 days post infection (dpi)) were treated with TNF- $\alpha(10 \mathrm{ng} / \mathrm{ml})$ for 16 hours or PMA (100 ng/ml)/Ionomycin (1 $\mu \mathrm{M})$ for $24 \mathrm{hours}(\mathrm{e})$ and VPA (indicated concentrations) for 48 hours (f). The Venus MFI (left) and Venus(+) population size (right) are shown $(\mathrm{n}=3$, mean \pm SD).

Involvement of PRC2 in establishing HIV-1 latency. HIV-1 integration preferentially occurs at active chromatin sites $^{32,33}$, where transcription is dynamically regulated by PRC2-mediated chromatin conformation. The results of ChIP assay and reactivation study implicated the involvement of PRC2 in the early phase of HIV-1 transcription silencing (Fig. 3). We performed knockdown experiments using shRNAs against PRC2 components EZH2 and SUZ12 to gain mechanistic insight into the contribution of PRC2 in the induction of LTR silencing. We tested previously validated shRNA sequences and selected one shRNA that showed the highest knockdown efficiency (Fig. 4a). Durable gene suppression was achieved by retrovirus-mediated stable shRNA expression. Jurkat cells were first transduced with shRNA-expressing retrovirus to establish stable knockdown and then infected with the reporter virus (Fig. 4b-g). Flow cytometry plots with sequential gating with
FSC/SSC and mRFP/Venus profiles revealed that the population size of the Venus(-) cells declined in the SUZ12- and EZH2-knockdowned or DZNep pre-treated cells (Fig. 4c-d). Alu-PCR showed almost the same amount of integrated LTR in these cells (Fig. 4e). ChIP assay also demonstrated the reduction of $\mathrm{H} 3 \mathrm{~K} 27 \mathrm{me} 3$ level on LTR region in the PRC2-depleted cells, suggesting rapid accumulation of the repressive histone modification in a part of infected population (Fig. 4f). A continuous culture revealed that PRC2 depletion induced higher LTR activity at the early time point (4 dpi) and inhibited time-dependent LTR silencing (Fig. 4g). Thus, PRC2 is suggested to be involved in rapid formation of the latently infected population.

To validate these results based on the new reporter construct, we employed a single-round HIV-1, which is a more practical model of HIV-1 infection. The molecular clone pNLnGFP-Kp, in which env 
a

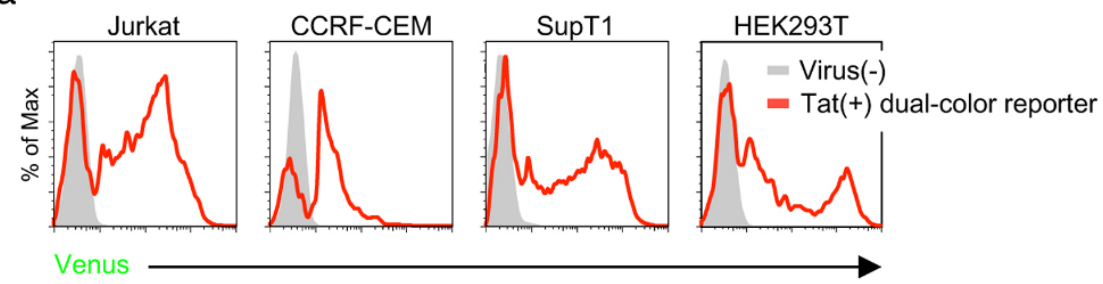

b

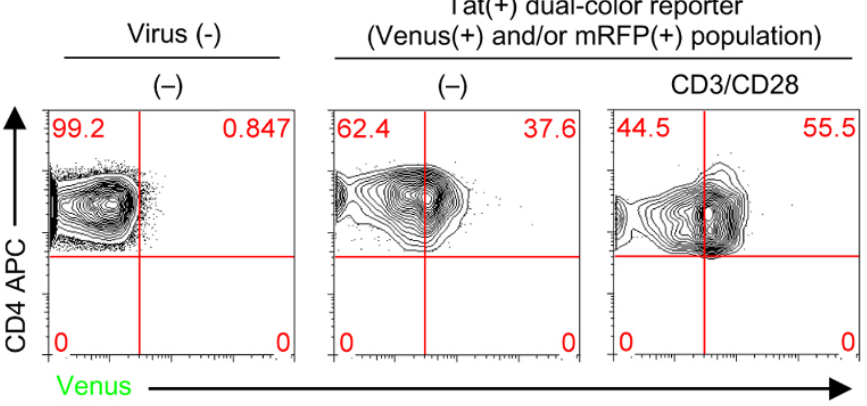

Figure $2 \mid$ LTR activity at establishment phase of HIV-1 infection. (a) Jurkat, CCRF-CEM, and SupT1 human T cell lines and HEK293T cells were infected with LTR-Tat-IRES-Venus-EF1 $\alpha-m R F P$ virus. The LTR activity within infected population $(\mathrm{mRFP}(+)$ and/or Venus $(+))$ was evaluated by flow cytometry at $3 \mathrm{dpi}$. (b) CD4+ T cells from a healthy donor were infected with LTR-Tat-IRES-Venus-EF1 $\alpha-m R F P$ virus. Flow cytometry plots represent Venus level within live, CD4 $(+)$, and $\mathrm{mRFP}(+)$ and/or Venus $(+)$ cells, based on the FSC/SSC and fluorescence profiles of the complete culture at $3 \mathrm{dpi}$. T cell activation was accomplished by treating the anti-CD3/CD28 antibodies for 3 days. Data are representative of three independent experiments.

contains a frame shift mutation, encodes EGFP in the nef region, enabling measurement of the LTR activity by the detection of EGFP fluorescence (Fig. 5a). EGFP activity reached a peak at 3 dpi and then decreased during culture under optimum conditions mainly because of viral cytotoxic effects (Fig. 5b). However, re-expression of EGFP was induced by treatment with TNF- $\alpha$ or HDAC inhibitor TSA at $14 \mathrm{dpi}$, suggesting the existence of latently infected cells in the EGFP $(-)$ populations (Fig. $5 \mathrm{c}$ ). This result also suggested that an epigenetic mechanism contributes to the LTR silencing, because HDAC inhibition could effectively reactivate LTR. We found that inhibition of EZH2 by DZNep in this population could also induce EGFP expression, indicating that the small population epigenetically remained as a latent population (Fig. 5c). Intriguingly, $\mathrm{T}$ cell activation could reactivate the $\mathrm{EGFP}(+)$ population at 3 days post singleround virus infection (Fig. $5 \mathrm{~d}-\mathrm{e}$ ). The reactivatable population size in PMA/Ionomycin treatment was larger than that of the EGFP expressing population at 4 days under normal cultivation, suggesting that silent integration occurs at the early time point of HIV-1 infection (Fig. 5e).

To address the PRC2 function in latency establishment in HIV-1 infection, we performed EZH2 and SUZ12 knockdown prior to infection of the single-round HIV-1 (Fig. 5f). Similar infectivity of HIV-1 was confirmed by Alu-PCR (Fig. 5g). Transition of EGFP expression revealed that the LTR activity was higher in EZH2- or SUZ12-knockdowned cells than the control cells (Fig. 5h). Furthermore, pre-treatment with DZNep and VPA showed similar results (Fig. 5h-j). Collectively, the results suggested that PRC2 negatively regulates the LTR activity immediately after integration of HIV-1, which may contribute to the establishment of silent integration.

Epigenetic suppression in actively integrated population. After infection of the reporter virus, the LTR activity in the actively infected population was gradually reduced during long-term culture (Figs. 1d and 6a). At $60 \mathrm{dpi}$, actively Venus expressing population was significantly decreased, resulting in substantial silencing of the LTR. ChIP assay revealed progressive gain of suppressive histone methylations in $\mathrm{H} 3 \mathrm{~K} 27$ and $\mathrm{H} 3 \mathrm{~K} 9$ during establishment of global latency (Fig. 6b). In addition, loss of the
$\mathrm{H} 3 \mathrm{Ac}$ marker indicated an epigenetic shift in transcriptional silencing. In contrast, the status of DNA methylation at the LTR region did not change over a long period (data not shown). Indeed, treating the SAHA or DZNep clearly prevented LTR silencing in the originally LTR active population (Fig. 6c). We further addressed the usefulness of a next generation EZH2 inhibitor, GSK $126^{34}$, in HIV-1 reactivation. GSK126 can specifically inhibit EZH2 activity. As a result, the GSK126-treated culture retained the Venus $(+)$ population (Fig. $6 \mathrm{c}$ ). Taken together, our results of the new reporter virus demonstrated that the transcriptional silencing of HIV-1 is established by two distinct mechanisms. A part of the infected cells possess epigenetically silenced LTR from the beginning of the infection. HIV-1 with a residual population is also epigenetically suppressed during long period. We found a significant difference in sensitivity against exogenous stimulation between the two distinctly infected populations. We compared the time-dependent silenced population from active infection (Venus $(+)$ population) and the originally silenced population (Venus $(-)$ population) by stimulating with TNF- $\alpha$ or PMA/Ionomycin. As a result, the LTR in the gradually silenced population was easily reactivated by exogenous stimulation. In contrast, the originally silenced LTR showed low sensitivity (Fig. 6d). We also treated the distinct population cultures with DZNep, VPA, or SAHA. As results, both populations could be significantly reactivated by the epigenetic drug combination (Fig. 6e). Of note, the time-dependent suppressed LTR was more susceptible than the immediately silenced population. GSK126 treatment in latently LTR also resulted in effective restoration of the LTR activity in both populations (Fig. 6e). These data collectively suggest that the heterogeneous mechanisms of HIV-1 silencing exist; one of them is established immediately after integration. Another mechanism is gradually formed in a timedependent manner and is more susceptible to signal activation or epigenetic reconstruction. Histone modifications appear to be one common molecular mechanism. We further confirmed LTR reactivation in the latently infected resting $\mathrm{CD} 4+\mathrm{T}$ cell population by $\mathrm{T}$ cell activation signal and epigenetic drugs (Fig. 6f). 
a
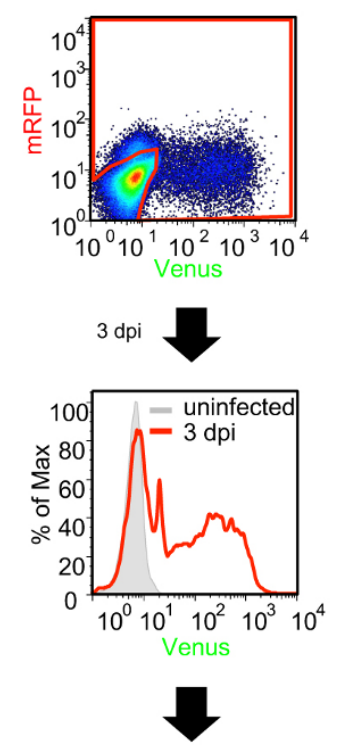

Cell sorting

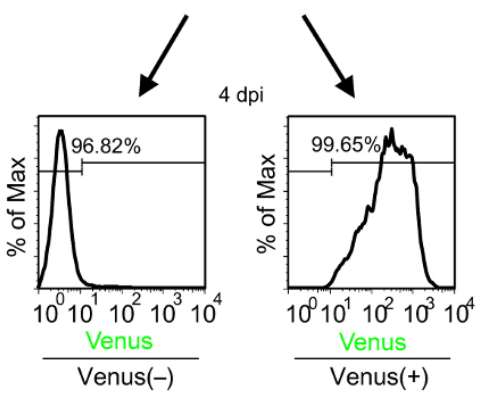

b

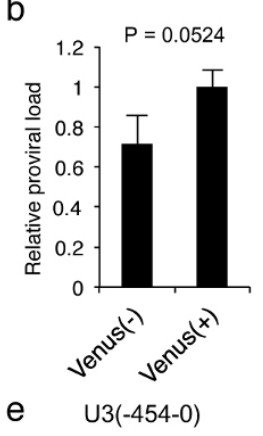

C
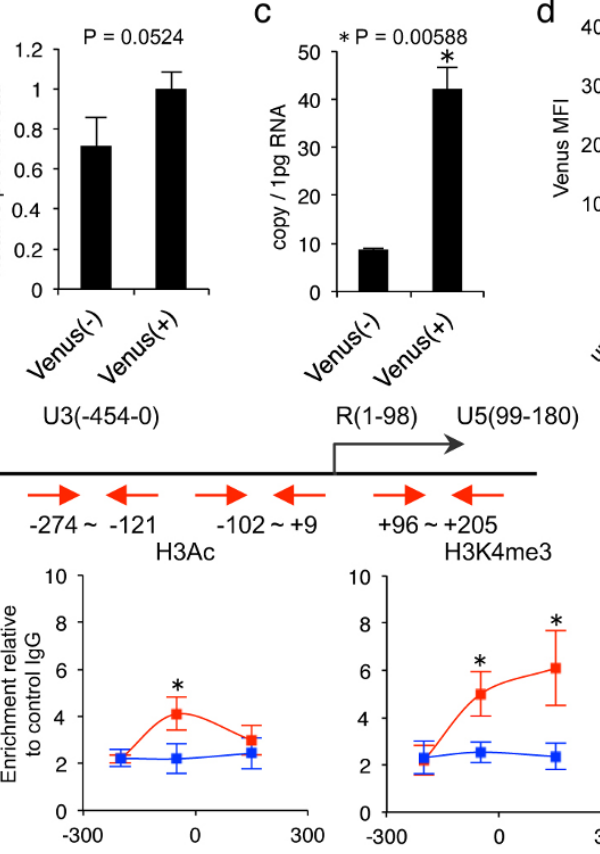

$\mathrm{f}$

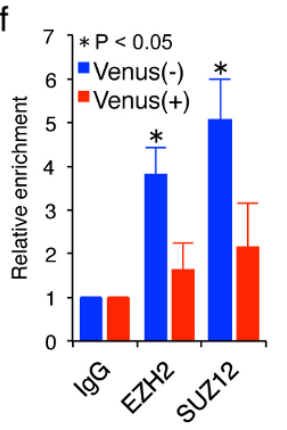

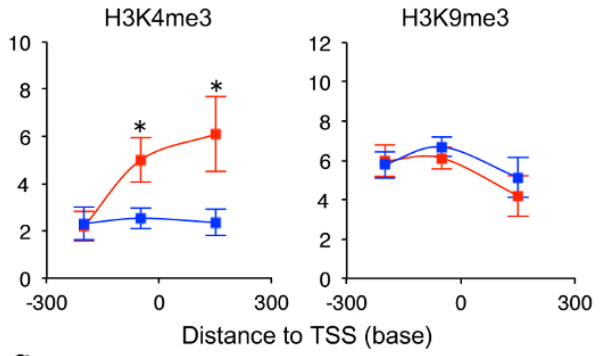

g

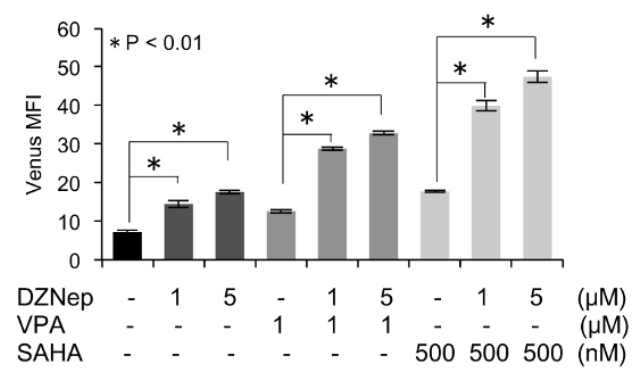

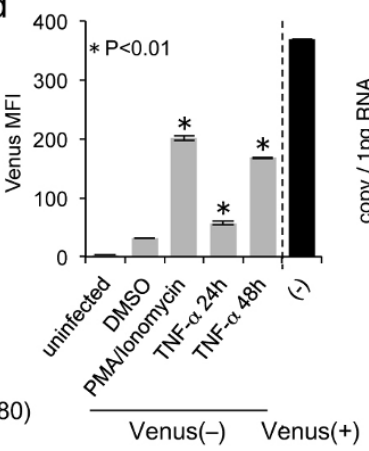

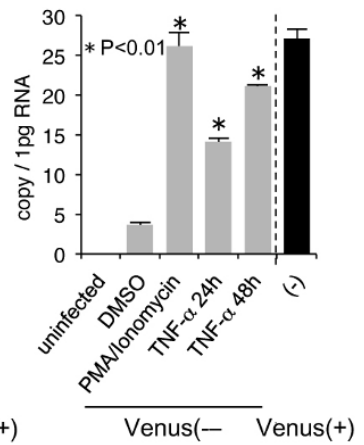

Venus(- Venus(+)

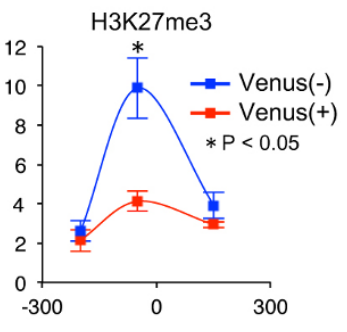

h

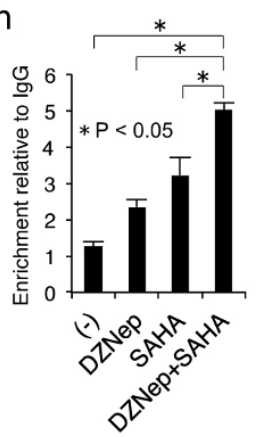

Figure 3 Epigenetic heterogeneity in HIV-1-infected sub-population. (a) An experimental strategy to investigate molecular basis of LTR silencing. Jurkat cells infected with the LTR-Tat-IRES-Venus-EF1 $\alpha-m R F P$ were sorted based on Venus and mRFP fluorescences at 3 dpi. The post-sorted populations were cultured individually and then subjected into molecular analyses $(\mathrm{n}=3$, representative data). (b) Proviral load from the both infected populations (10 dpi) was evaluated by Alu-PCR method $(\mathrm{n}=3$, mean $\pm \mathrm{SD}$ ). Albumin DNA was also analyzed as an internal standard. (c) Venus mRNA amount was absolutely quantified in Venus $(-)$ and Venus $(+)$ populations $(\mathrm{n}=3$, mean $\pm \mathrm{SD})$. $\beta$-actin mRNA level was measured as an internal control. (d) The Venus(-) population (20 dpi) was treated with PMA/Ionomycin (24 hours) or TNF- $\alpha$ (24 and 48 hours). Venus MFI (left) and absolute Venus mRNA amount (right) were determined $(\mathrm{n}=3$, mean $\pm \mathrm{SD})$. (e) ChIP assay. Histone covalent modifications at LTR region in the sorted Venus $(+)$ and Venus(-) sub-populations were analyzed by PCR-based ChIP assay at 13 dpi. Positions of primer sets for the real-time PCR are indicated in upper diagram. $\mathrm{H} 3$ acetylation (AcH3), H3K4 trimethylation (H3K4me3), H3K9 trimethylation (H3K9me3), and H3K27 trimethylation (H3K27me3) were analyzed using specific antibodies. Enrichment values relative to control IgG and LTR positions are plotted $(\mathrm{n}=3$, mean $\pm \mathrm{SD}$ ). (f) The two distinct populations ( $13 \mathrm{dpi}$ ) were subjected in ChIP assay with anti-EZH2, anti-SUZ12, and control antibodies $(\mathrm{n}=3$, mean $\pm \mathrm{SD})$. (g,h) Venus $(-)$ population (20 dpi) was treated with indicated dose of PRC2 inhibitor DZNep and HDAC inhibitors VPA and SAHA for 48 hours. The reactivated Venus MFI (g) and CTD-phosphorylated Pol II occupancy (h) were evaluated by flow cytometry and ChIP assay, respectively $(\mathrm{n}=3$, mean \pm SD).

Polycomb-mediated epigenetic silencing of HIV-1. The results of the new reporter virus prompted us to address the PRC2 contribution in the establishment of HIV-1 latency. Therefore, we utilized two additional latency models, Hela/LTR-luciferase and $\mathrm{U} 1$ cells, to analyze the PRC2 function in the regulation of HIV-1 latency. Knockdown of the PRC2 factors, EZH2 and SUZ12, released the LTR activity in HeLa cells (Fig. 7a-b). In addition, SUZ12 depletion led to increased sensitivity against the Tat protein (Fig. 7c) and vice versa. Indeed, enforced SUZ12 expression by plasmid transfection suppressed Tat-dependent LTR transactivation (Fig. 7d). Similar results were obtained from the $\mathrm{U} 1$ cell, which was chronically infected with HIV $-1^{35}$. Knockdown of EZH2 or SUZ12 altered histone methylation levels at the promoter region of host MYT1, whose expression was constantly regulated by a PRC2-mediated epigenetic mechanism (Fig. 7e-f). In these PRC2-knockdowned cells, the H3K27me3 mark at the LTR region was substantially diminished (Fig. 7g). Viral gene transcription was activated in EZH2- and SUZ12-knockdown cells. These cells showed high sensitivity against TNF- $\alpha$ stimulation, suggesting that PRC2dependent histone methylation significantly affected viral gene regulation (Fig. 7h). Depletion of PRC2 function did not have any effects on other histone markers. Collectively, PRC2 is a host restriction complex at the establishment and maintenance of LTR silencing by regulating epigenetic states in the LTR region.

\section{Discussion}

So far, many researchers have developed original models to investigate the mechanism of HIV-1 latency and revealed several molecular states of the stably silenced $5^{\prime}$ LTR sequence ${ }^{36}$. However, a detailed mechanism in the establishment step of the transcriptional dorm- 

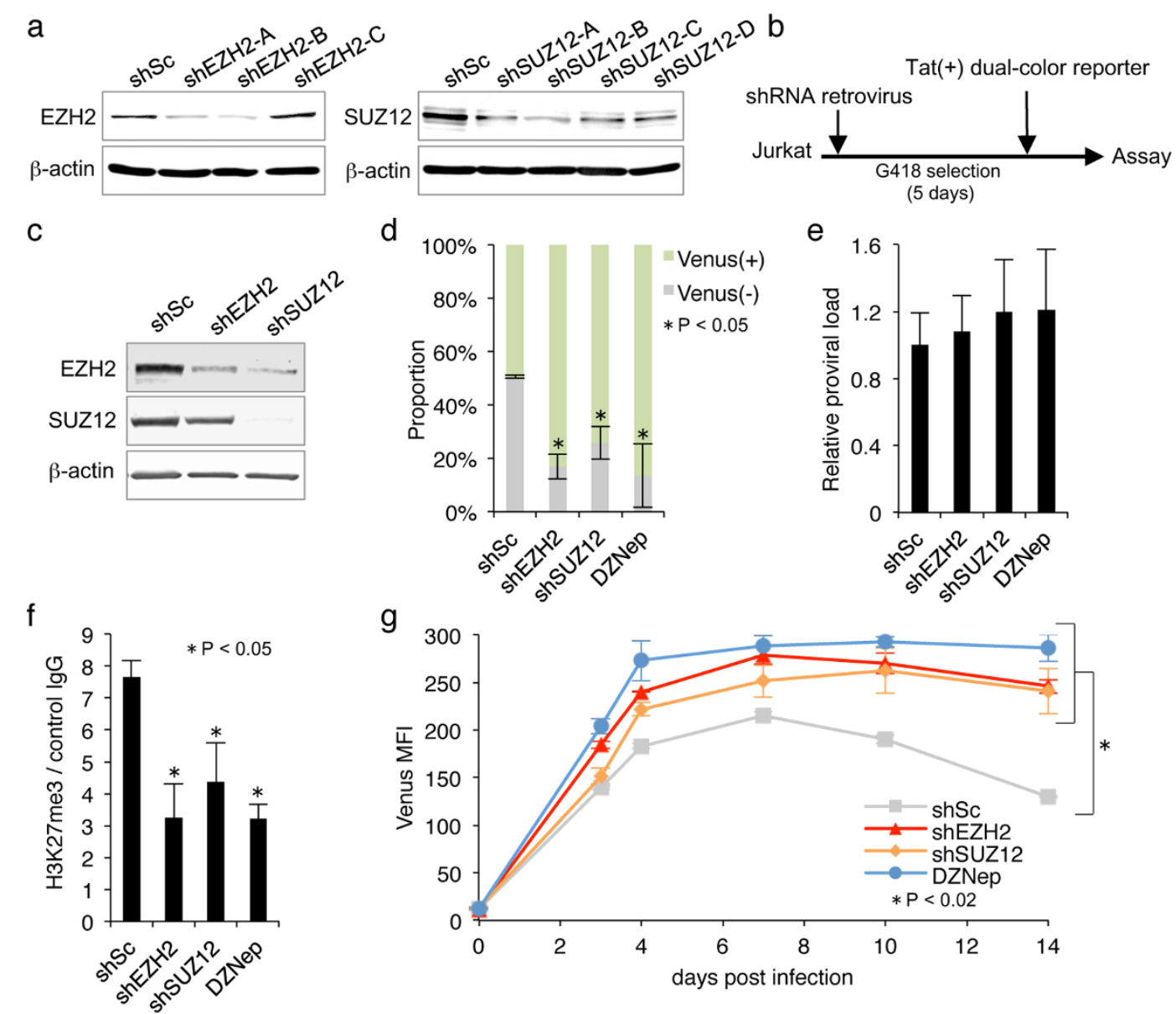

Figure 4 Involvement of PRC2 in silencing establishment. (a) Previously confirmed shRNAs targeting EZH2 and SUZ12 were introduced in Jurkat cells by retrovirus vectors. Western blots showed knockdown efficiencies of the EZH2 (left) and SUZ12 (right). shSc indicates a scrambled control shRNA ${ }^{32}$. $\beta$ actin was evaluated as an internal control. Original data are presented in Supplementary Figure 2. shEZH2-A and shSUZ12-A are mainly used for the following experiments. (b) An experimental procedure to examine the role of PRC2 in HIV-1 silencing. Prior to the dual-color reporter infection, knockdown of PRC2 factors was accomplished by retrovirus-mediated shRNA expression. The Tat $(+)$ dual-color reporter virus was then inoculated into the EZH2- or SUZ12-depleted Jurkat cells. (c) Knockdown efficiencies of EZH2 and SUZ12 were confirmed by Western blots. Original data are presented in Supplementary Figure 2. (d) Proportional size of Venus $(+) / \operatorname{Venus}(-)$ were determined at 4 dpi by flow cytometry $(\mathrm{n}=3$, mean \pm SD). (e) Proviral load from the PRC2-depleted infected populations ( $4 \mathrm{dpi}$ ) evaluated by Alu-PCR ( $\mathrm{n}=3$, mean \pm SD). (f) H3K27me3 level on LTR region at 4dpi ( $\mathrm{n}=3$, mean $\pm \mathrm{SD})$. (g) The shRNA-expressing and reporter-infected cells were continuously cultured and profiled the Venus/mRFP expression. Venus MFI within infected population was plotted for $14 \mathrm{dpi}(\mathrm{n}=3$, mean $\pm \mathrm{SD})$.

ancy is not fully understood, because of the low frequency of cells harboring silenced HIV-1 and lack of methods to detect such populations in the early phase of HIV-1 infection. In this study, we developed a new reporter virus that carries two different fluorescent genes downstream in LTR and constitutive promoter EF1 $\alpha$, enabling us to analyze the dynamic regulation of the LTR activity. It also allowed us to address a series of epigenetic changes in a fixed, integrated LTR sequence because of the absence of new infection and toxicity from viral gene expression. The Alu-PCR method ensured proviral integration in the interested populations. Using the unbiased model, we identified two ways of transcriptional repression of HIV-1, i.e., expeditious disappearance and continuous decline of HIV-1 gene expression. Surprisingly, one infected population showed very low LTR activity immediately after infection in various cell types, including $\mathrm{T}$ cell lines and primary $\mathrm{CD} 4+\mathrm{T}$ cells (Fig. 2). NL4-3 strain-derived single-round HIV-1 also produced a similar LTR-repressed population at the early phase of $\mathrm{T}$ cell infection (Fig. 5). However, the other population presented strong LTR promoter activity immediately after infection, which was gradually suppressed during long-term cultivation (Fig. 6).

Accumulated evidence suggests that host epigenetic factors appear to restrict gene expression from HIV-1 provirus and its propagation in chronically infected individuals ${ }^{26,37}$. However, mechanistic insight into the establishment of transcriptional latency has not been implicated because most previous studies have used stably silenced LTR models. Making use of the traits of the new dual-color reporter mentioned above, we could visualize the epigenetic heterogeneity in LTR at the latency establishment step. Evaluation of covalent histone markers revealed that trimethylation at $\mathrm{H} 3 \mathrm{~K} 27$ was significantly accumulated at the early phase of infection, particularly the promoter region of LTR in the quickly silenced population. H3K9 trimethylation, which involved transcriptional repression, was not different between the two populations at the early point. H3K27 methylation is catalyzed by PRC2 factors including EZH2, SUZ12, and EED. Recently, Friedman et al. reported the involvement of EZH2 in the maintenance of HIV-1 silencing by using their latent model Jurkat E4 clone ${ }^{38}$. It has been noted that the HIV-1 provirus is preferentially integrated in the euchromatic region where basal transcription activity is dynamically regulated by PRC2-mediated H3K27me3 ${ }^{32,39,40}$. We prevented PRC2 function by treating with DZNep or the novel EZH2 inhibitor GSK126 and could elevate LTR activity from the immediate silent population (Fig. 3g and 6e). shRNA-directed EZH2 depletion prior to LTR integration resulted in an imbalance of immediate silent and active infections 
a

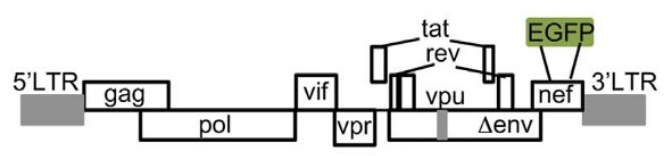

C

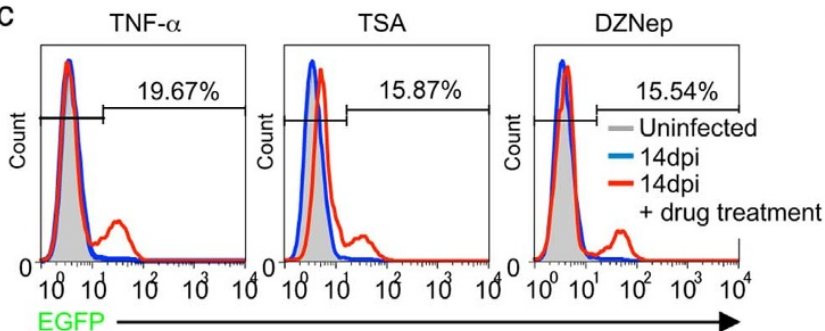

b

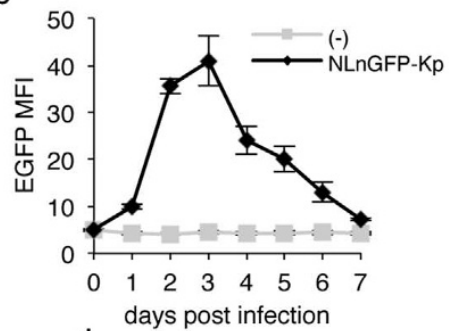

d

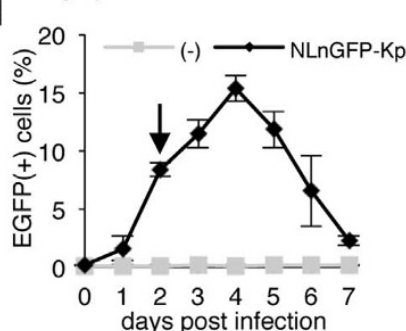

e

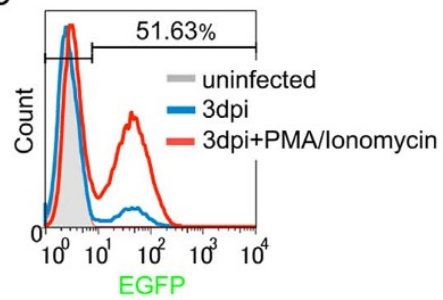

f

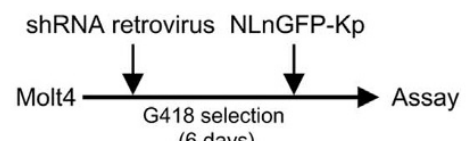

g

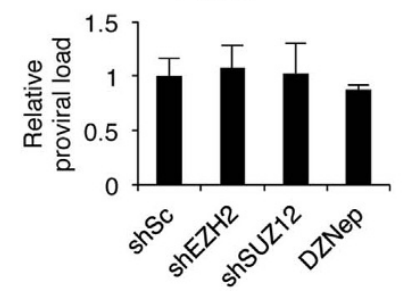

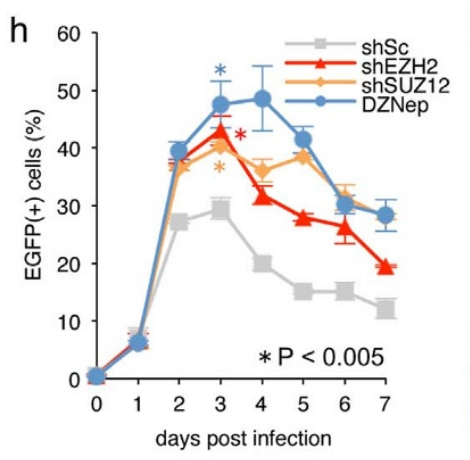

i

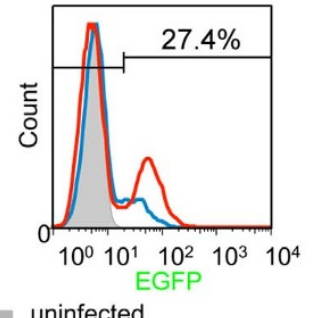

- uninfected

- DMSO pretreatment HIV(+)

- VPA pretreatment $\operatorname{HIV}(+)$ j

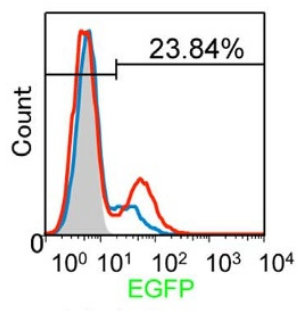

- uninfected

- DMSO pretreatment HIV(+)

- DZNep pretreatment HIV(+)

Figure $5 \mid$ Latency establishment in single-round HIV-1. (a) Proviral structure of a single-round HIV-1 pNLnGFP-Kp ${ }^{31}$. The concentrated, VSV-Gpseudotyped virus could be effectively introduced in targeted T cell lines. The LTR activity could be directly monitored by EGFP detection. (b) Molt4 T cell line was infected with the single-round HIV-1. After washout of residual viruses, the cells were continuously cultured under the normal condition. The EGFP MFI was determined by flow cytometry $(\mathrm{n}=3$, mean \pm SD). (c) Molt4 cells infected with the single-round HIV-1 were treated with TNF- $\alpha$ (10 ng/ $\mathrm{ml}, 16$ hours), TSA (500 nM, 16 hours), or DZNep ( $5 \mu \mathrm{M}, 48$ hours) at 14 dpi. The reactivated population size was defined as EGFP positivity. Data are representative of three independent experiments. (d,e) CCRF-CEM cells were infected with the single-round HIV-1 and analyzed the EGFP $(+)$

population size $(\mathrm{n}=3$, mean $\pm \mathrm{SD})(\mathrm{d})$. Arrow indicates time point of drug treatment. The infected cells ( $2 \mathrm{dpi})$ were treated with PMA (100 ng/ml)/ Ionomycin $(1 \mu \mathrm{M})$ for 24 hours. The EGFP expression pattern was determined by flow cytometry at 3 dpi (Data are representative of three independent experiments) (e). ( $\mathrm{f}-\mathrm{h}$ ) Influence of PRC2 pre-inhibition. Molt4 cells were infected with the shRNA-expressing retroviruses prior to infection of the single-round HIV-1 (f). The viral DNA was quantified by Alu-PCR at 2 dpi ( $\mathrm{n}=3$, mean $\pm \mathrm{SD}$ ) (g). After the single-round HIV-1 infection, the EGFP expression was continuously examined by flow cytometry $(n=3$, mean \pm SD) $(h)$. (i,j), Jurkat cells were pre-treated with VPA (i) and DZNep $(j)$ for 48 hours and then infected with the single-round HIV-1. EGFP expression levels were evaluated at 3 dpi (Data are representative of three independent experiments).

(Fig. 4). The fact that the decline of SUZ12 led to the same result indicated the importance of the complex-dependent epigenetic manner in the immediate silencing. ChIP assay with EZH2 and SUZ12 antibodies demonstrated occupancy of the PRC2 factor at transcription start site (TSS) in LTR (Fig. 3f). Collectively, our data suggest that PRC2-dependent epigenetic control of LTR is involved in the establishment of immediate silent infection. In contrast, silencingassociated DNA methylation was not detected in our model. Importantly, we also observed that major active histone modifications, H3K4 trimethylation and histone acetylation, were significantly higher in the actively infected population than the immediately silenced population (Fig. 3e). Indeed, HDAC inhibitors such as VPA and SAHA, which are implicated as new drugs to purge latently infected cells ${ }^{41}$, could reactivate LTR. Of note, cooperation of histone acetylation and H3K27me3 appeared to coordinate the actual LTR activity (Fig. 3g, 6e-f). Further study about H3K4me3 and its regulator trithorax group, which is a cellular counterpart of polycomb, may help to understand the molecular basis of the early step of HIV-1 regulation.
Time-dependent regression of the LTR activity has certainly been implicated. Because this reporter does not express toxic viral genes and expresses fluorescent proteins enabling us to purify the populations involved, we addressed the dynamic course of LTR repression in the actively infected population. Expectedly, the LTR activity within the active population gradually reduced during culture under normal conditions. Interestingly, ChIP assay revealed that $\mathrm{H} 3 \mathrm{~K} 27 \mathrm{me} 3$ accumulated at the LTR region in the time-dependent silenced population was similar to the immediately silent population (Fig. 6b). These data suggest that PRC2-mediated H3K27me3 and its negative gene regulation may contribute to HIV-1 silencing between the early phase establishment and late phase maintenance of HIV-1 latency. Whereas, the acquisition of $\mathrm{H} 3 \mathrm{~K} 9 \mathrm{me} 3$, which has already been implicated in HIV-1 latency ${ }^{42,43}$, appears to be involved in late stage maintenance of HIV-1 latency. Importantly, LTR responses to epigenetic drugs and exogenous stimuli clearly differed in the two distinct populations. We found that the repressed LTR in the originally active infection population was highly sensitive not only to NF$\kappa \mathrm{B}$ stimulators but also to epigenetic drugs compared with the 

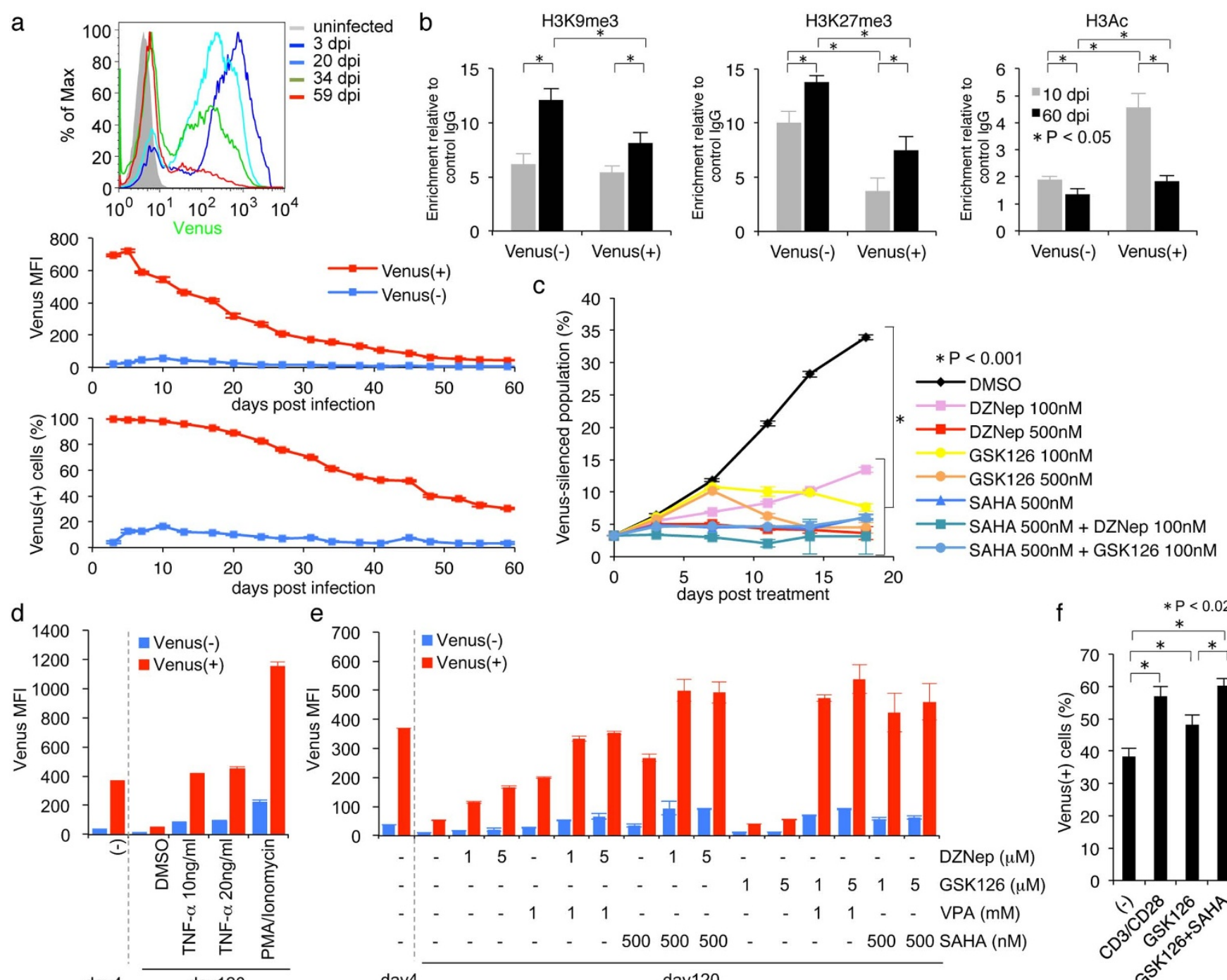

e
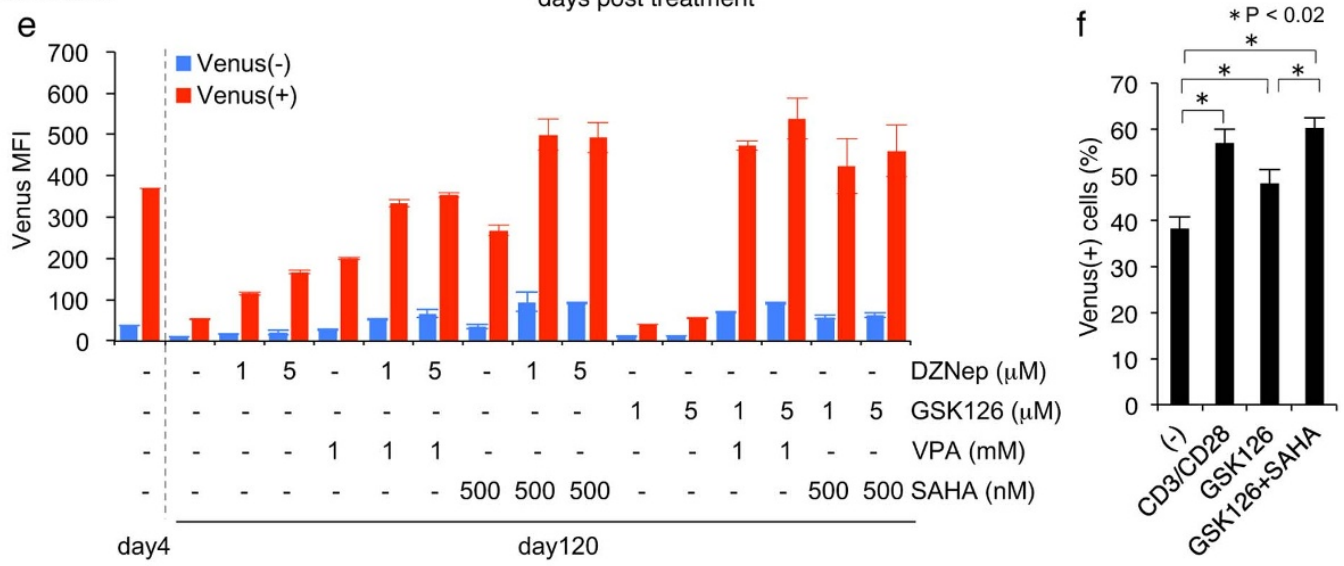

Figure 6 | LTR silencing in actively infected cells. (a) Jurkat cells were infected with the LTR-Tat-IRES-Venus-EF1 $\alpha-m R F P$ virus. The Venus $(+)$ population was sorted based on the Venus expression at 3 dpi and cultured individually for 60 days. LTR activity was evaluated by Venus expression level. The flow cytometry histograms (upper histogram) and the progress of MFI (middle graph) and proportion of Venus $(+)$ population (lower graph) are presented (red line). The progress of immediately silenced population is also depicted (blue line) ( $\mathrm{n}=3$, mean \pm SD). (b) ChIP assay with indicated antibodies at 10 and 60 dpi revealed changes of the histone modifications at the LTR promoter region in the immediately silenced population (shown as $\operatorname{Venus}(-))$ and the time-dependent silenced population (shown as Venus $(+)(\mathrm{n}=3$, mean \pm SD). (c) The sorted Venus $(+)$ population at 7 dpi was treated with the epigenetic drug combination for 18 days. The transition of Venus expression was examined by flow cytometry. The population size of Venus-silenced cells was plotted $(\mathrm{n}=3$, mean $\pm \mathrm{SD})$. $(\mathrm{d}, \mathrm{e})$ The time-dependent silenced population from active infection $($ Venus $(+)$, red bar) and the originally silenced population (Venus(-), blue bar) were treated with TNF- $\alpha$ or PMA/Ionomycin for 24 hours (d) and DZNep, VPA, or SAHA for 48 hours or GSK126 for 96 hours (e) at 120 dpi. The LTR activity was evaluated by Venus MFI $(\mathrm{n}=3$, mean \pm SD). (f) Resting CD4+ T cells were infected with the dual-color reporter virus and then reactivated with indicated drugs for 3 days. Venus $(+)$ population size within the infected cells at 6 dpi were shown $(\mathrm{n}=3$, mean $\pm \mathrm{SD})$.

immediately silent population (Fig. 6d-e). In addition to the epigenetic regulation, other mechanisms such as integration site preferences or differences in host factor expression could be associated with determining the robustness of LTR latency ${ }^{44-48}$. Of late, noninduced HIV-1 provirus was identified in clinical samples ${ }^{49}$. The strong suppression appeared to occur by transcriptional dormancy, leading to an underestimation of latent reservoir size based on a $\mathrm{T}$ cell activation survey. The immediately silenced population that we identified in this study might explain this concept. By combining our reporter system with massively parallel DNA sequencing or comprehensive expression profiling, we will be able to clarify the residual question in the near future.

In the context of an epigenetic maintenance in LTR regulation, components of the polycomb family appear to be categorized as host restriction factors because SUZ12 overexpression inhibited Tatmediated LTR activation, and vice versa in stable latent models (Fig. 7). PRC2 has central roles in the regulation of expression of a number of host genes ${ }^{50}$. We recently reported that aberrant activation of PRC2 leads to microRNA suppression, which in turn activates $\mathrm{NF}-\kappa \mathrm{B}$ signaling in $\mathrm{T}$ cell $\mathrm{s}^{51}$. Understanding the accurate regulatory mechanism and function of PRC2 in T cells may be helpful for intervention of latent HIV-1. It is also necessary to understand the molecular mechanisms of how PRC2 is recruited to LTR in the latency population. Recruiters of mammalian PRC2 appear to be locus specific and still largely unknown. In addition, one major challenge is to delineate the role of Tat in epigenetic regulation of LTR. Absence of Tat creates robust latent population (Fig. 1). Because Tat fluctuation drives phenotypic diversity ${ }^{52}$, interplay between host epi- 


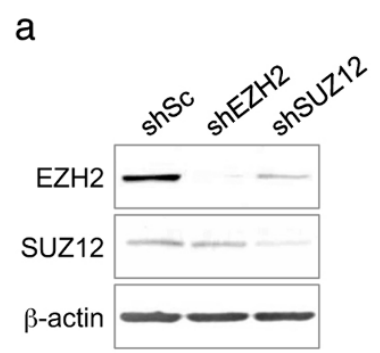

e

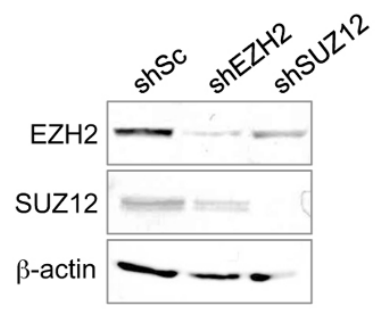

b

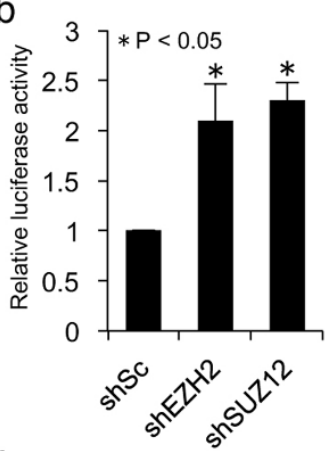

f

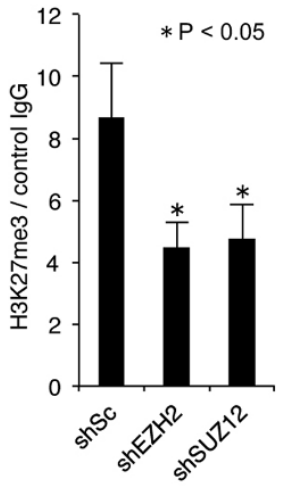

C

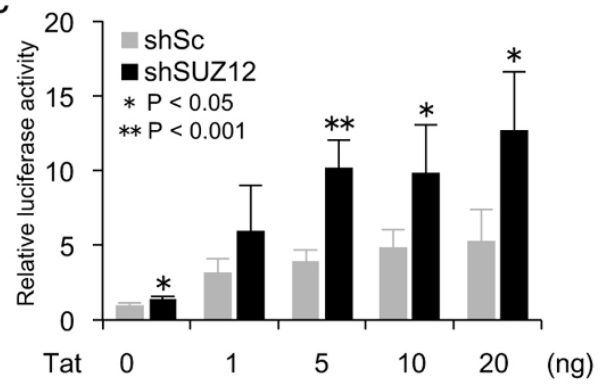

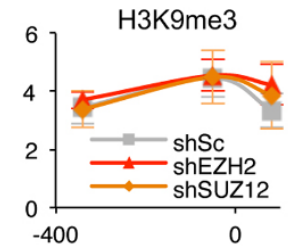

d

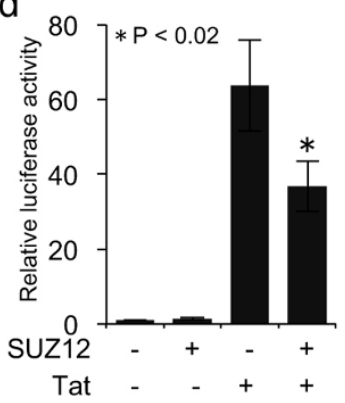

$\mathrm{h}$

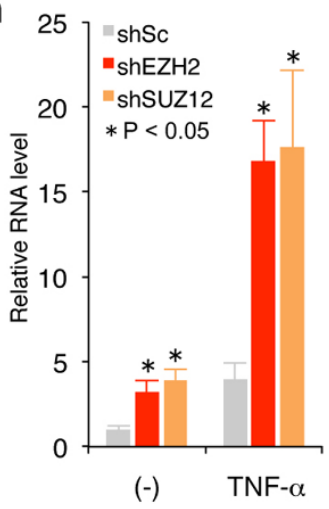

Figure 7 Role of PRC2 in LTR regulation. (a-d) PRC2 contributes to LTR silencing in HeLa/LTR-luciferase cells. HeLa/LTR-luciferase cells were infected with the shSUZ12-, shEZH2-, or scrambled shRNA (shSc)-expressing retrovirus. Knockdown efficiency was confirmed by Western blots (a, original data are presented in Supplementary Figure 2). The LTR activities of the knockdowned cells were examined by luciferase assay $(\mathrm{n}=3$, mean \pm SD) (b). HeLa/LTR-luciferase cells with shSUZ12 or shSc were transfected with various dose of Tat expression plasmid DNA. The luciferase activity was evaluated at 24 hours post transfection $(n=6$, mean \pm SD) $(c)$. HeLa/LTR-luciferase cells were transfected with the plasmid DNA encoding Tat or SUZ12. The luciferase activity was examined at 24 hours post Tat transfection $(\mathrm{n}=6$, mean \pm SD) (d). (e-h) PRC2 contributes to HIV-1 silencing in U1 cells. The U1 cells were infected with the retrovirus expressing shEZH2, shSUZ12, or shSc. (e) Western blots confirmed depletion of EZH2 or SUZ12. Original data are presented in Supplementary Figure 2. (g,f) ChIP assay showed PRC2 knockdown-associated alterations of the H3K27me3 level at MYT1 promoter region and the various histone modifications at LTR region $(\mathrm{n}=3$, mean $\pm \mathrm{SD})$. (h) Relative gag RNA levels in shRNA-expressing U1 cells in the presence or absence of TNF- $\alpha(10 \mathrm{ng} / \mathrm{ml}, 48$ hours $)(\mathrm{n}=3$, mean $\pm \mathrm{SD})$.

genetic factors and viral factors may dominate the integrated LTR activity.

Resting CD4 + T cells are largely non-permissive for HIV-1 replication because of high activity of triphosphohydrolase SAMHD $1^{53}$ and rigid actin cortex barrier ${ }^{54}$. To simply examine the dynamic activity and epigenetic state of LTR integrated in the quiescent primary CD4 $+\mathrm{T}$ cells, we performed reporter infection in non-activated $\mathrm{CD} 4+\mathrm{T}$ cell by using the rigorously concentrated dual-color reporter virus, which is derived from single-round high-titer lentivirus system ${ }^{55}$. Spinoculation method ${ }^{56}$ facilitates viral entry and following integration in resting $\mathrm{CD} 4+\mathrm{T}$ cell. Figure $2 \mathrm{~b}$ have shown the heterogeneity of LTR activity in resting CD $4+\mathrm{T}$ cells. It clearly reflected that number of cells having Venus expression increased upon $\mathrm{CD} 3 / \mathrm{CD} 28$ stimulation, indicating that many cells are productive as compared to the resting stage. There are many articles, which support this observation in vitro and in vivo.

Taken together, results from studies with new reporter and some latent models have illustrated that PRC2 plays key roles in the establishment and maintenance of virus transcriptional repression. The distinct ways of infection produced at least two latently infected populations. Although the clinical importance of these populations is still open to question, our findings raise the interesting possibility that PRC2 depletion reduces the size of the deeply inactivated virus population (Fig. 4). Furthermore, co-treatment with PRC2 and HDAC inhibitors more effectively restored the silenced LTR activity in both cell line models and resting CD4 + T cells. Our observations suggest that PRC2 inhibition may be a valuable future strategy to prevent establishment of HIV-1 silencing and eliminate latent reservoirs, which is an urgent challenge in HIV studies. Because epigenetic markers are potentially reversible, genuine epigenetic-targeted therapy may hold great promise for HIV-1 eradication.

\section{Methods}

Cell culture and drug treatments. Jurkat, Molt4, CCRF-CEM, SupT1 and U1 cells were maintained in RPMI1640 medium (GIBCO) with 10\% fetal bovine serum (FBS, GIBCO), and antibiotics (GIBCO). For exogenous stimulation, the cells were treated with PMA (LC Laboratories), Ionomycin (CALBIOCHEM), or TNF- $\alpha$ (R\&D systems). For inhibition of epigenetic factors, the cells were treated with the indicated concentrations of VPA (SIGMA), SAHA (Cayman), TSA (SIGMA), 3deazaneplanocin A (DZNep, Cayman), or GSK126 (Chemie Tek). HEK293T, HEK293FT, and Hela/LTR-luciferase cells were maintained in DMEM with 10\% FBS and antibiotics. Human peripheral blood mononuclear cells (PBMCs) were prepared from whole blood of healthy donors by density gradient centrifugation with FicollPaque (GE healthcare). CD4 + T cells were purified by CD4+ T cell isolation kit (Miltenyi Biotec) and maintained in RPMI1640 with $10 \%$ of FBS. T cell activation was accomplished by treating the anti-CD3/CD28 antibodies (Miltenyi Biotec).

Generation and transduction of dual-color reporter virus. For generation of the dual-color reporter lentivirus DNA, Replication-defective, self-inactivating lentivirus vectors CSII-EF-MCS-IRES2-Venus and CS-RfA-EF-mRFP1 were used ${ }^{55}$. DNA primers used for generating the vectors are described in supplementary information. HIV-1 LTR sequence was obtained from genomic DNA of HIV-1 NL4-3-infected Molt 4 cells by PCR amplification with a primer set (AgeI LTR forward and AgeI LTR reverse). The amplified LTR DNA was inserted into an EF1 $\alpha$ promoter region of the CSII-EF-MCS-IRES2-Venus plasmid. Tat cDNA from HIV-1 NL4-3 was amplified by PCR with a primer set (NotI Tat forward and BamHI Tat reverse) and inserted into NotI and BamHI sites within CSII-LTR-MCS-IRES2-Venus plasmid. The established LTR-Tat/empty-IRES2-Venus sequence was amplified by PCR with primers (SalI LTR-Tat-IRES-Venus For and XbaI LTR-Tat-IRES-Venus Rev), 
followed by cloning into pENTR4-H1 vector at SalI and XbaI sites. The produced entry vector was recombined with a CS-RfA-EF-mRFP1 vector by Gateway LR Clonase II Enzyme Mix (Invitrogen).

For production of reporter virus, the accomplished lentiviral plasmid vector (CSLTR-Tat/empty-IRES-Venus-EF-mRFP) was co-transfected with the packaging plasmids (pCAG-HIVgp and pCMV-VSV-G-RSV-Rev) into 293FT cells. High-titer viral solutions were prepared by centrifugation-based concentration at $8,000 \times g$ for at least 3 hours and used for transduction into target cells. $1 \times 10^{6}$ cells were infected with the lentivirus by spinoculation method (1,800 rpm, 2 hours) and then cultured in $2 \mathrm{ml}$ of RPMI 1640 with $10 \%$ FCS. For primary CD $4+$ T cells, approximately $20 \mathrm{ml}$ of VSV-G-pseudotyped reporter virus solution from the transfection supernatant were concentrated by centrifugation and used for $1 \times 10^{6}$ primary $\mathrm{T}$ cells.

Single-round HIV infection. A single-round HIV molecular clone pNLnGFP-Kp ${ }^{57}$ was provided from Prof. A. Adachi, Tokushima University, Japan. The plasmid and the VSV-G- and Rev-expressing vector were co-transfected into 293FT cells. At 48 hours post-transfection, the culture supernatant was $0.45 \mu \mathrm{m}$ filtrated and concentrated by centrifugation at $8,000 \times g, 3$ hours at $4^{\circ} \mathrm{C}$. The collected virus solution was immediately used or kept in $-80^{\circ} \mathrm{C}$ freezer.

Flow cytometric analysis. Expression levels of florescent proteins were analyzed by FACSCalibur (BD). The reporter-introduced cells were harvested and re-suspended in $0.3 \% \mathrm{FBS} / \mathrm{PBS}$. LTR activity in the infected population was defined by calculating the expression levels of Venus (FL1) and mRFP (FL3). For EGFP detection, the singleround HIV-infected cells were fixed by $3 \%$ formaldehyde before flow cytometry. $\mathrm{CD} 4+\mathrm{T}$ cells were defined by staining with APC-conjugated anti-Human CD4 antibody (BD, 551980)

Chromatin immunoprecipitation (ChIP) assay. Histone covalent modifications were quantified by PCR-ChIP assay ${ }^{58}$ with some modifications. Briefly, the cells $(1 \times$ $10^{6}$ ) were cross-linked with $1 \%$ formaldehyde for $15 \mathrm{~min}$ at room temperature, washed with PBS at $4{ }^{\circ} \mathrm{C}$, and suspended in SDS-lysis buffer (50 mM Tris-HCl, $1 \%$ SDS, 10 mM EDTA, $\mathrm{pH}$ 8.1, protease inhibitor cocktail). The lysate was then sonicated on ice for $1 \mathrm{~min} 40 \mathrm{sec}$ (Astreson ultrasonic processor, MISONIX Inc.). After centrifugation, the supernatants were subjected to immunoprecipitation with specific antibodies; anti-H3K27me3 (Active Motif, \#39155), anti-H3K9me3 (Abcam, \#ab8898-100), anti-H3K4me3 (Cell Signaling, \#9751S), anti-AcH3 (Millipore, \#06599), anti-EZH2 (Cell Signaling, \#5246S), anti- SUZ12 (Cell Signaling, \#3737S), antiCTD-phosphorylated Pol II (COVANCE, MMS-134R), anti-normal Rabbit IgG (Cell Signaling, \#2729S). The precipitated DNA was purified and analyzed by real-time PCR with specific primers (supplementary information).

Retroviral shRNA knockdown. shRNA-expressing retroviral vector (pSINsi-U6, TAKARA, Japan) was used for EZH2 and SUZ12 knockdowns. The specific retroviral vectors were established by insertion of synthesized oligonucleotides using BamHI and $\mathrm{ClaI}$ sites. Sequences of the inserted oligonucleotides have previously been tested (supplementary information). Retrovirus production was carried out in 293T cells with the pSIN vector, pGP vector ( $g a g-p o l$ coding, TAKARA), and pCMV-VSV-GRSV-Rev. At 48 hours post-transfection, the retrovirus solution was $0.45 \mu \mathrm{m}$ filtrated, concentrated by centrifugation, and added to target cells for 24 hours establishment. The transduced cells were selected with $0.5 \mathrm{mg} / \mathrm{ml}$ of G418 for 5-6 days.

Western blotting. Whole cell lysates were analyzed by 7.5 to $10 \%$ acrylamide Tris$\mathrm{HCl}$ buffered SDS-PAGE. Western blots were performed with first antibodies listed below; $\beta$-actin (SantaCruz Biotechnology, sc-8432), EZH2 (Cell Signaling, \#3147S), SUZ12 (Cell Signaling, \#3737S). Alkaline phosphatase-conjugated anti-mouse or anti-rabbit IgG was used as a secondary antibody. BCIP/NBT substrate (Promega) with ALP buffer (100 mM Tris- $\mathrm{HCl}$ pH 9.5, $\left.100 \mathrm{mM} \mathrm{NaCl}, 50 \mathrm{mM} \mathrm{MgCl}_{2}\right)$ was used for detection.

PCR. Total RNA isolation was performed using ISOGEN (Wako, Japan). The DNaseI-treated total RNA was subjected to reverse-transcriptase (RT) reaction using SuperScript II (Invitrogen) with manufacturer's protocol. The oligo dT primersbased synthesized cDNA was analyzed by quantitative PCR using real-time PCR system (Thermal cycler Dice, TAKARA). The specific PCR was performed using gene-specific primers and SYBRGreen (TAKARA). The level of $\beta$-actin was quantified for internal control. Absolute copy number was determined by standard curve method. Genomic DNA in each infected population was extracted using QIAamp DNA Blood Mini Kit (QIAGEN). Alu-based PCR was performed to quantify integrated HIV-1 provirus, as previously described ${ }^{59}$.

Reporter gene assay. HeLa/LTR-luciferase cells were previously established ${ }^{58}$. The firefly luciferase activity was analyzed by luciferase reporter assay system (Promega) and normalized by protein concentration of cell lysate.

1. Perelson, A. S. et al. Decay characteristics of HIV-1-infected compartments during combination therapy. Nature 387, 188-191 (1997).

2. Gulick, R. M. et al. Treatment with indinavir, zidovudine, and lamivudine in adults with human immunodeficiency virus infection and prior antiretroviral therapy. N. Engl. J. Med. 337, 734-739 (1997).
3. Finzi, D. et al. Identification of a reservoir for HIV-1 in patients on highly active antiretroviral therapy. Science 278, 1295-1300 (1997).

4. Wong, J. K. et al. Recovery of replication-competent HIV despite prolonged suppression of plasma viremia. Science 278, 1291-1295 (1997)

5. Chun, T. W., Davey, R. T., Jr, Engel, D., Lane, H. C. \& Fauci, A. S. Re-emergence of HIV after stopping therapy. Nature 401, 874-875 (1999).

6. Chun, T. W. et al. Presence of an inducible HIV-1 latent reservoir during highly active antiretroviral therapy. Proc. Natl. Acad. Sci. U.S.A. 94, 13193-13197 (1997).

7. Bailey, J. R. et al. Residual human immunodeficiency virus type 1 viremia in some patients on antiretroviral therapy is dominated by a small number of invariant clones rarely found in circulating CD4+ T cells. J. Virol. 80, 6441-6457 (2006).

8. Kieffer, T. L. et al. (2004) Genotypic analysis of HIV-1 drug resistance at the limit of detection: virus production without evolution in treated adults with undetectable HIV loads. J. Infect. Dis. 189, 1452-1465 (2004).

9. Frenkel, L. M. et al. Multiple viral genetic analyses detect low-level human immunodeficiency virus type 1 replication during effective highly active antiretroviral therapy. J. Virol. 77, 5721-5730 (2003).

10. Kinoshita, S., Chen, B. K., Kaneshima, H. \& Nolan, G. P. Host control of HIV-1 parasitism in T cells by the nuclear factor of activated T cells. Cell 95, 595-604 (1998).

11. Perkins, N. D. et al. A cooperative interaction between NF-kappa B and Sp1 is required for HIV-1 enhancer activation. EMBO J. 12, 3551-3558 (1993).

12. Kim, Y. K., Mbonye, U., Hokello, J. \& Karn, J. T-cell receptor signaling enhances transcriptional elongation from latent HIV proviruses by activating P-TEFb through an ERK-dependent pathway. J. Mol. Biol. 410, 896-916 (2011).

13. Kao, S. Y., Calman, A. F., Luciw, P. A. \& Peterlin, B. M. Anti-termination of transcription within the long terminal repeat of HIV-1 by tat gene product. Nature 330, 489-493 (1987).

14. Wei, P., Garber, M. E., Fang, S. M., Fischer, W. H. \& Jones, K. A. A novel CDK9associated C-type cyclin interacts directly with HIV-1 Tat and mediates its highaffinity, loop-specific binding to TAR RNA. Cell 92, 451-462 (1998).

15. Van Lint, C., Emiliani, S., Ott, M. \& Verdin, E. Transcriptional activation and chromatin remodeling of the HIV-1 promoter in response to histone acetylation. EMBO J. 15, 1112-1120 (1996).

16. Williams, S. A. et al. NF-kappaB p50 promotes HIV latency through HDAC recruitment and repression of transcriptional initiation. EMBO J. 25, 139-149 (2006).

17. Tyagi, M. \& Karn, J. CBF-1 promotes transcriptional silencing during the establishment of HIV-1 latency. EMBO J. 26, 4985-4995 (2007).

18. Jiang, G., Espeseth, A., Hazuda, D. J. \& Margolis, D. M. c-Myc and Sp1 contribute to proviral latency by recruiting histone deacetylase 1 to the human immunodeficiency virus type 1 promoter. J. Virol. 81, 10914-10923 (2007).

19. Cohen, J. Exploring how to get at--and eradicate--hidden HIV. Science $\mathbf{2 7 9}$ 1854-1855 (1998)

20. Chun, T. W., Engel, D., Mizell, S. B., Ehler, L. A. \& Fauci, A. S. Induction of HIV-1 replication in latently infected CD4+ T cells using a combination of cytokines. J. Exp. Med. 188, 83-91 (1998).

21. Chun, T. W. et al. Effect of interleukin-2 on the pool of latently infected, resting $\mathrm{CD} 4+\mathrm{T}$ cells in HIV-1-infected patients receiving highly active anti-retroviral therapy. Nat. Med. 5, 651-655 (1999).

22. Davey, R. T., Jr. et al. HIV-1 and T cell dynamics after interruption of highly active antiretroviral therapy (HAART) in patients with a history of sustained viral suppression. Proc. Natl. Acad. Sci. U.S.A. 96, 15109-15114 (1999).

23. Stellbrink, H. J. et al. Effects of interleukin-2 plus highly active antiretroviral therapy on HIV-1 replication and proviral DNA (COSMIC trial). AIDS 16, 1479-1487 (2002).

24. Lafeuillade, A. et al. Pilot study of a combination of highly active antiretroviral therapy and cytokines to induce HIV-1 remission. J. Acquir. Immune Defic. Syndr. 26, 44-55 (2001)

25. Lehrman, G. et al. Depletion of latent HIV-1 infection in vivo: a proof-of-concept study. Lancet 366, 549-555 (2005)

26. Archin, N. M. et al. Administration of vorinostat disrupts HIV-1 latency in patients on antiretroviral therapy. Nature 487, 482-485 (2012)

27. Ruelas, D. S. \& Greene, W. C. An integrated overview of HIV-1 latency. Cell 155 , 519-529 (2013)

28. Chun, T. W. et al. Quantification of latent tissue reservoirs and total body viral load in HIV-1 infection. Nature 387, 183-188 (1997).

29. Ylisastigui, L., Archin, N. M., Lehrman, G., Bosch, R. J. \& Margolis, D. M. Coaxing HIV-1 from resting CD4 T cells: histone deacetylase inhibition allows latent viral expression. AIDS 18, 1101-1108 (2004).

30. Ishida, T., Hamano, A., Koiwa, T. \& Watanabe, T. 5' long terminal repeat (LTR)selective methylation of latently infected HIV-1 provirus that is demethylated by reactivation signals. Retrovirology 3, 69 (2006).

31. Blazkova, J. et al. CpG methylation controls reactivation of HIV from latency. PLoS Pathog. 5, e1000554 (2009).

32. Schroder, A. R. et al. HIV-1 integration in the human genome favors active genes and local hotspots. Cell 110, 521-529 (2002).

33. Han, Y. et al. Resting CD4+ T cells from human immunodeficiency virus type 1 (HIV-1)-infected individuals carry integrated HIV-1 genomes within actively transcribed host genes. J. Virol. 78, 6122-6133 (2004).

34. McCabe, M. T. et al. EZH2 inhibition as a therapeutic strategy for lymphoma with EZH2-activating mutations. Nature 492, 108-112 (2012). 
35. Emiliani, S. et al. Mutations in the tat gene are responsible for human immunodeficiency virus type 1 postintegration latency in the U1 cell line. J. Virol. 72, 1666-1670 (1998).

36. Colin, L. \& Van Lint, C. Molecular control of HIV-1 postintegration latency: implications for the development of new therapeutic strategies. Retrovirology 6 , 111 (2009).

37. Archin, N. M. et al. Expression of latent HIV induced by the potent HDAC inhibitor suberoylanilide hydroxamic acid. AIDS Res. Hum. Retroviruses. 25 207-212 (2009).

38. Friedman, J. et al. Epigenetic silencing of HIV-1 by the histone $\mathrm{H} 3$ lysine 27 methyltransferase enhancer of Zeste 2. J. Virol. 85, 9078-9089 (2011).

39. Kouzarides, T. Chromatin modifications and their function. Cell 128, 693-705 (2007)

40. Barski, A. et al. High-resolution profiling of histone methylations in the human genome. Cell 129, 823-837 (2007).

41. Geeraert, L., Kraus, G. \& Pomerantz, R. J. Hide-and-seek: the challenge of viral persistence in HIV-1 infection. Annu. Rev. Med. 59, 487-501 (2008).

42. du Chene, I. et al. Suv39H1 and HP1gamma are responsible for chromatinmediated HIV-1 transcriptional silencing and post-integration latency. EMBO J. 26, 424-435 (2007).

43. Marban, C. et al. Recruitment of chromatin-modifying enzymes by CTIP2 promotes HIV-1 transcriptional silencing. EMBO J. 26, 412-423 (2007).

44. Duverger, A. et al. Determinants of the establishment of human immunodeficiency virus type 1 latency. J. Virol. 83, 3078-3093 (2009).

45. Burnett, J. C., Miller-Jensen, K., Shah, P. S., Arkin, A. P. \& Schaffer, D. V. Control of stochastic gene expression by host factors at the HIV promoter. PLoS Pathog. 5, e1000260 (2009)

46. Duverger, A. et al. An AP-1 binding site in the enhancer/core element of the HIV1 promoter controls the ability of HIV-1 to establish latent infection. J. Virol. 87, 2264-2277 (2013).

47. Tyagi, M., Pearson, R. J. \& Karn, J. Establishment of HIV latency in primary $\mathrm{CD} 4+$ cells is due to epigenetic transcriptional silencing and $\mathrm{P}-\mathrm{TEFb}$ restriction. J. Virol. 84, 6425-6437 (2010).

48. Budhiraja, S., Famiglietti, M., Bosque, A., Planelles, V. \& Rice, A. P. Cyclin T1 and CDK9 T-loop phosphorylation are downregulated during establishment of HIV-1 latency in primary resting memory CD4+ T cells. J. Virol. 87, 1211-1220 (2013).

49. Ho, Y. C. et al. Replication-competent noninduced proviruses in the latent reservoir increase barrier to HIV-1 cure. Cell 155, 540-551 (2013).

50. Kirmizis, A. et al. Silencing of human polycomb target genes is associated with methylation of histone H3 Lys 27. Genes Dev. 18, 1592-1605 (2004).

51. Yamagishi, M. et al. Polycomb-mediated loss of miR-31 activates NIK-dependent NF-kappaB pathway in adult T cell leukemia and other cancers. Cancer Cell 21, 121-135 (2012).

52. Weinberger, L. S., Burnett, J. C., Toettcher, J. E., Arkin, A. P. \& Schaffer, D. V. Stochastic gene expression in a lentiviral positive-feedback loop: HIV-1 Tat fluctuations drive phenotypic diversity. Cell 122, 169-182 (2005).

53. Baldauf, H. M. et al. SAMHD1 restricts HIV-1 infection in resting CD4(+) T cells. Nat. Med. 18, 1682-1687 (2012).
54. Pan, X., Baldauf, H. M., Keppler, O. T. \& Fackler, O. T. Restrictions to HIV-1 replication in resting CD4+ T lymphocytes. Cell Res. 23, 876-885 (2013).

55. Miyoshi, H., Takahashi, M., Gage, F. H. \& Verma, I. M. Stable and efficient gene transfer into the retina using an HIV-based lentiviral vector. Proc. Natl. Acad. Sci. U.S.A. 94, 10319-10323 (1997).

56. O’Doherty, U., Swiggard, W. J. \& Malim, M. H. Human immunodeficiency virus type 1 spinoculation enhances infection through virus binding. J. Virol. 74, 10074-10080 (2000).

57. Fukumori, T. et al. Regulation of cell cycle and apoptosis by human immunodeficiency virus type 1 Vpr. Microbes Infect. 2, 1011-1017 (2000).

58. Yamagishi, M. et al. Retroviral delivery of promoter-targeted shRNA induces long-term silencing of HIV-1 transcription. Microbes Infect. 11, 500-508 (2009).

59. Brussel, A. \& Sonigo, P. Analysis of early human immunodeficiency virus type 1 DNA synthesis by use of a new sensitive assay for quantifying integrated provirus. J. Virol. 77, 10119-10124 (2003).

\section{Acknowledgments}

We thank Drs. H. Miyoshi and A. Miyawaki for providing the Venus- and mRFP-encoding lentivirus vectors. This work is supported by JSPS KAKENHI Grant Number 24790436 (M.Y.), 23390250 (T.W.), Grant-in-Aid from the Ministry of Health, Labour and Welfare H24-AIDS-008 (T.W.), and grant from the Uehara Memorial Foundation (M.Y.).

\section{Author contributions}

Y.M. and M.Y. performed the all experiments. M.K.I., D.F. and T.I. advised the experimental design and protocols used in this study. T.W. and M.Y. conceived the study, designed the experiments and helped in drafting and finalizing the manuscripts. All authors reviewed the manuscript.

\section{Additional information}

Supplementary information accompanies this paper at http://www.nature.com/ scientificreports

Competing financial interests: The authors declare no competing financial interests.

How to cite this article: Matsuda, Y. et al. Epigenetic Heterogeneity in HIV-1 Latency Establishment. Sci. Rep. 5, 7701; DOI:10.1038/srep07701 (2015).

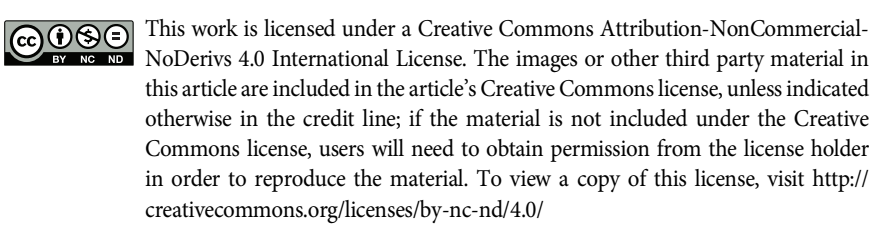

